\title{
A Simplified Nonlinear Sway-Rocking Model for Evaluation of Seismic Response of Structures on Shallow Foundations
}

\author{
Yang $\mathrm{Lu}^{1^{*}}$, Alec M. Marshall ${ }^{1}$, Iman Hajirasouliha ${ }^{2}$ \\ ${ }^{1}$ Department of Civil Engineering, University of Nottingham, University Park, Nottingham NG7 2RD, UK \\ ${ }^{2}$ Department of Civil \& Structural Engineering, University of Sheffield, Western Bank Sheffield S10 2TN, UK \\ *Corresponding Author: E-mail: evxyl7@nottingham.ac.uk
}

\begin{abstract}
This paper presents a simplified nonlinear sway-rocking model as a preliminary design tool for seismic soil-structure interaction analysis. The proposed model is intended to capture the nonlinear load-displacement response of shallow foundations during strong earthquake events where foundation bearing capacity is fully mobilized. Emphasis is given to heavily-loaded structures resting on a saturated clay half-space. The variation of soil stiffness and strength with depth, referred to as soil non-homogeneity, is considered in the model. Although independent springs are utilized for each of the swaying and rocking motions, coupling between these motions is taken into account by expressing the load-displacement relations as functions of the factor of safety against vertical bearing capacity failure (FSV) and the moment-to-shear ratio $(M / H)$. The simplified model has been calibrated and validated against results from a series of static push-over and dynamic analyses performed using a more rigorous finite-difference numerical model. Despite some limitations of the current implementation, the concept of this model gives engineers more degrees of freedom in defining their own model components, providing a good balance between simplicity, flexibility and accuracy.
\end{abstract}

Keywords: Soil-structure interaction; Simplified model; Nonlinear analysis; Soil non-homogeneity; Coupled sway-rocking response 


\section{Nomenclature}

\begin{tabular}{|c|c|}
\hline$A$ & Area of the mat foundation \\
\hline c & Shape parameter of the backbone curve used in NSR model \\
\hline$C_{r}$ & Initial elastic range of the backbone curve used in NSR model \\
\hline$D$ & Diameter of the mat foundation \\
\hline$E_{f}$ & Young's modulus of the foundation material \\
\hline$F_{0}$ & Force at the start of the current plastic loading cycle \\
\hline$F_{c}$ & Bearing capacity of foundation under combined loading \\
\hline$F_{\text {in }}$ & Force when first entering the plastic cycle \\
\hline FSv & Factor of safety of foundation against pure static vertical load \\
\hline$G$ & Soil shear modulus \\
\hline$G_{0}$ & Soil shear modulus at ground level \\
\hline$H$ & Foundation shear force \\
\hline$H_{c}$ & Foundation shear capacity under combined loading \\
\hline$H_{u}$ & Foundation shear capacity under pure sliding \\
\hline$h_{\text {eff }}$ & Effective height of the superstructure \\
\hline$h_{i}$ & Height of superstructure from base to the $\mathrm{i}^{\text {th }}$ level \\
\hline$h_{\text {tot }}$ & Total height of the superstructure \\
\hline$K$ & Bulk modulus \\
\hline$k_{h}$ & Foundation swaying stiffness \\
\hline$k_{h r}\left(k_{r h}\right)$ & Coupled term in foundation stiffness matrix \\
\hline$k_{\text {in }}$ & Initial foundation stiffness after vertical load is fully developed \\
\hline$k_{n}$ & Normal stiffness of the interface \\
\hline$k_{r}$ & Foundation Rocking stiffness \\
\hline$k_{s}$ & Tangential stiffness of the interface \\
\hline$M$ & Foundation rocking moment \\
\hline$M_{c}$ & Foundation moment capacity under combined loading \\
\hline$M_{u}$ & Foundation moment capacity under pure rocking \\
\hline$m$ & Mass of the superstructure \\
\hline$N$ & Number of storeys \\
\hline$N_{c M}$ & Foundation ultimate moment capacity coefficient \\
\hline$R$ & Radius of the mat foundation \\
\hline$s_{u}$ & Soil undrained shear strength \\
\hline$s_{u 0}$ & Soil undrained shear strength at ground level \\
\hline$u$ & Foundation sliding displacement \\
\hline$u_{50}$ & Total foundation displacement at which $50 \%$ of capacity is mobilized \\
\hline$u_{e}$ & Elastic component of foundation displacement \\
\hline$u_{p}$ & Plastic component of foundation displacement \\
\hline$u_{p o}$ & Plastic component of foundation displacement at the start of the current plastic loading cycle \\
\hline$V$ & Foundation vertical force \\
\hline$V_{u}$ & Foundation bearing capacity under pure vertical load \\
\hline$w$ & Foundation settlement \\
\hline$\rho$ & Mass density \\
\hline$\alpha_{h}$ & Stiffness loss factor for foundation swaying response \\
\hline$\alpha_{r}$ & Stiffness loss factor for foundation rocking response \\
\hline$\beta$ & Gradient defining the stiffness and strength profile of the foundation soil \\
\hline$\chi$ & Influence factor for foundation stiffness taking into account soil heterogeneity \\
\hline$\varphi_{i 1}$ & Amplitude at the $\mathrm{i}^{\text {th }}$ storey corresponding to the fundamental mode of vibration of the superstructure \\
\hline$\lambda$ & Soil non-homogeneity index \\
\hline$v$ & Poisson's ratio \\
\hline$v_{f}$ & Poisson's ratio of the foundation material \\
\hline$\theta$ & Foundation rotation \\
\hline
\end{tabular}




\section{Introduction}

During the past decade, the interest in the topic of seismic Soil-Structure Interaction (SSI) has seen a gradual shift from the superstructure to the foundation soil. Recent research studies on SSI have shown reduced seismic ductility demands of structures due to nonlinearity that arises mainly from the mobilization of the ultimate capacity and the uplifting response of shallow foundations. These studies have mainly focused on stiff slender structures on small foundations, such as shear walls [1], bridge piers [2, 3], and framed structures [4, 5] supported by spread footings. It has been found that the lifting off of one side of the footing not only results in geometric nonlinearity at the soil-footing interface, but causes yielding of soil on the other side, which in turn increases the uplift. Allowing mobilization of the foundation bearing capacity through soil yielding and foundation uplifting limits the maximum loads that can act on the superstructure, and also leads to a considerable amount of energy dissipation due to the hysteretic damping in the soil [6].

On the other hand, structures supported on spread footings may experience unexpectedly high differential settlements during strong shaking. This phenomenon, induced by either heavy structural loads that are unevenly distributed across the footing, poor soil conditions, or the combination of both, can lead to failure of structural components and hence, non-repairable damage or collapse of structures [7]. Mat (or Raft) foundations, in these cases, are more suitable to spread the loads from the structure to the ground. Unlike the shear walls or bridge piers, structures supported on mat foundations are usually designed with a medium slenderness ratio. This leads to a strong interaction between the sway and rocking motions of the foundation when subjected to the horizontal component of strong ground motion.

It has been shown that nonlinearities in the soil (corresponding to large strains) and at the soilfoundation interface are almost unavoidable in strong seismic events [8]. Performance-based seismic design methodology embraces these nonlinearities, provided that the responses of both structural and geotechnical components satisfy the performance targets. The role of nonlinear seismic soil-structure interaction on dynamic response of buildings has recently been emphasized by Pecker et al. [9] and Gazetas [10]. In this context, it is important to develop reliable design tools that provide sufficient accuracy to assess the seismic performance of SSI while maintaining simplicity so as to be easily understood and accepted by engineers.

In recent years, the concept of a macro-element, which simplifies the dynamic interaction between soil and foundation by integrating the nonlinearities (in the soil and/or at the soil-foundation interface) into a single plasticity-based element, has attracted considerable attention (e.g. [11-13]). However, this macro element for practical engineers remains a "black box" where the multi-yield (and sometimes multi-mechanism) complexity makes it difficult to be implemented into computer codes [14].

On the other hand, using spring-type models to simulate the dynamic response of soil-structure systems is popular in design practice because of their ease of use and clear physical meaning. Examples include (1) the linear dynamic impedance models (e.g. cone model [15]) used in the analysis of foundation vibrations on an elastic soil medium, (2) Winkler-based linear/nonlinear spring-bed models (e.g. [16, 17]), and (3) the nonlinear rotational spring model [18] for the analysis of rocking-dominant nonlinear foundation behaviour. These models usually assume that the foundation soil is homogeneous, whereas in most cases the soil stiffness and strength increase with 
depth due to the effects of overburden stress. There is a lack of an effective and efficient spring-type model which is able to capture both nonlinear sway-rocking response of shallow foundations and soil non-homogeneity.

This paper presents a simplified Nonlinear Sway-Rocking (NSR) model that is capable of simulating the load-displacement response of mat foundations subjected to seismic excitations. Compared with the linear/nonlinear spring-type models in the literature, the present model in this study is able to simulate the nonlinear foundation sway-rocking response which can be significantly affected by the load path of the seismically-excited SSI system. The effect of soil non-homogeneity is also considered. The model is developed using the OpenSees platform [23] and verified using data obtained from more rigorous Finite Difference (FD) analyses conducted using LLAC $^{3 \mathrm{D}}$. The simplified model is well suited for heavily-loaded structures with a moderate slenderness ratio for which the nonlinear sway response is strongly coupled with the rocking response.

The paper is organized into six main sections. First, an overview of the problem is provided, followed by a description of a FLAC ${ }^{3 D}$ numerical model and static analyses conducted to identify the foundation load-displacement relations and bearing capacities. The NSR model is then developed based on calibration of analytical foundation backbone curves with load-displacement relations obtained from the $\mathrm{FLAC}^{3 \mathrm{D}}$ static push-over tests. The process by which the coupling between swaying and rocking motions is taken into account in the proposed model is also described. The efficiency of the NSR model to predict load-displacement and moment-rotation responses of shallow foundations to dynamic loading is demonstrated using results obtained from additional dynamic $\mathrm{FLAC}^{3 \mathrm{D}}$ numerical simulations. Finally, the limitations of the model are discussed and conclusions are provided.

\section{Problem Definition}

The problem investigated in this study (Fig. 1) is a seismically-excited building founded on a halfspace consisting of saturated soft clay layers, where undrained shear strength $s_{u}$ and stiffness $G$ increase linearly with depth (Poisson's ratio $v$ and density $\rho$ remain constant). The foundation is assumed to be rigid, which is appropriate for a mat foundation that is much stiffer than the soil. Foundation movements are described by the translations $w$ (vertical) and $u$ (horizontal) as well as rotation angle $\theta$, which are correlated, respectively, with the forces $V, H$ and moment $M$ with respect to the base centre. The interface between the foundation and clay is assumed to sustain tension. This simplifying assumption is suitable for the problem being investigated: buildings supported on soft clay deposits and subjected to undrained seismic loading where the foundation uplift resistance is provided by structural weight and potential suction within the underlying soil. The overall SSI system is initially subjected to the self-weight $V$ of the structure, followed by the radial load paths in the $M-H$ plane representing the seismically loaded structures with a predominant mode of vibration. Fig. 1(b) displays the simplified NSR model where the mass of the structure $m$ is lumped at an effective height $h_{\text {eff }}$ above a circular mat foundation with an equivalent radius $R=$ $\sqrt{A / \pi}$, where $A$ is the area of the foundation. In the proposed model, the soil half-space is replaced by an assemblage of springs and dashpots. The plastic spring (placed closer to the foundation) simulates the large-displacement behaviour of the soil-foundation system, whereas the spring and dashpot arranged in parallel are used to model, respectively, the small-displacement response and the radiation damping. The strength and stiffness of the springs and the damping coefficient of the 
dashpots were derived from existing analytical solutions and rigorous FD analyses. Though independent springs are used for the individual degrees of freedom of the foundation, coupling between the nonlinear sway and rocking responses is also incorporated in the model by expressing the spring properties as a function of the load paths experienced by the foundation in $V: H: M$ space. The load paths are controlled by the factor of safety against vertical bearing capacity failure FSv, the moment-to-shear ratio $M / H$, and the failure envelope defining the ultimate limit states of the foundation in $V: H: M$ space.

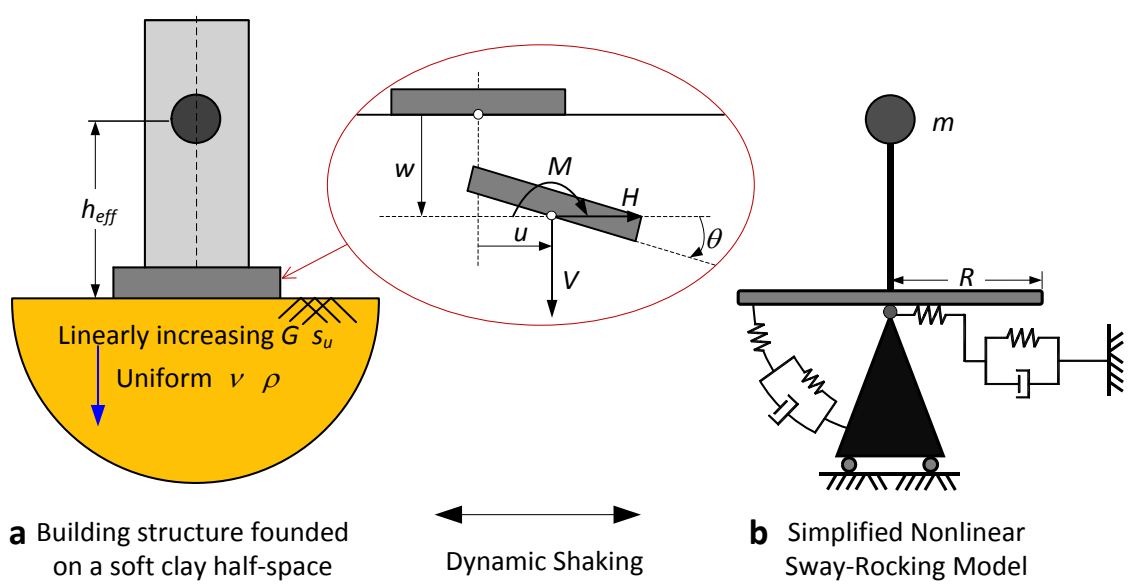

Fig. 1. Problem illustration: (a) a seismically-excited building supported by a shallow foundation resting on a soil half-space; and (b) simplified nonlinear sway-rocking model consisting of an assemblage of springs and dashpots for simulating the seismic behaviour of the soil-foundation system.

\section{Finite Difference Model (Static Analysis)}

A set of numerical analyses was performed using $\mathrm{FLAC}^{3 D}$ [19] in order to obtain data for the calibration of the NSR model. FLAC ${ }^{3 D}$ is a three-dimensional finite-difference program that was developed for applications in soil and rock mechanics and has been used widely for both static and dynamic problems. In this study, the standard linear elastic perfectly plastic model using the Tresca failure criterion (total stress analysis) and an associated flow rule were utilized to represent saturated clay behaviour under undrained conditions.

\subsection{FLAC $^{3 \mathrm{D}}$ Model}

As shown in Fig. 2, the FLAC ${ }^{3 \mathrm{D}}$ model represents a circular foundation of diameter $D$ resting on the surface of a cylindrical stratum of saturated clay defined by the undrained shear strength $s_{u}$, the shear modulus $G=500 s_{u}$, and Poisson's ratio $v=0.49$. Taking advantage of symmetry, only half of the model was considered in the analytical modelling. The undrained shear strength was assumed to increase linearly with depth, which results in a profile described by:

$$
s_{u}=s_{u 0}+\beta z
$$

where $s_{u 0}$ is the shear strength at ground level and $\beta$ is the strength gradient with depth $z$. Note that $G$ also increases at a gradient $\beta$ with depth, leading to a generalized "Gibson" type stiffness profile [20]. The degree of non-homogeneity is measured by a dimensionless number [21]: 


$$
\lambda=\frac{\beta D}{s_{u 0}}
$$

with $\lambda$ increasing from 0 to 6 , indicating a transition from homogeneous to increasingly heterogeneous soil conditions. In this study, three values of $\lambda$ were considered, with $\lambda=0,2$ and 6 representing homogeneous, moderately non-homogeneous and highly non-homogeneous soil conditions, respectively.

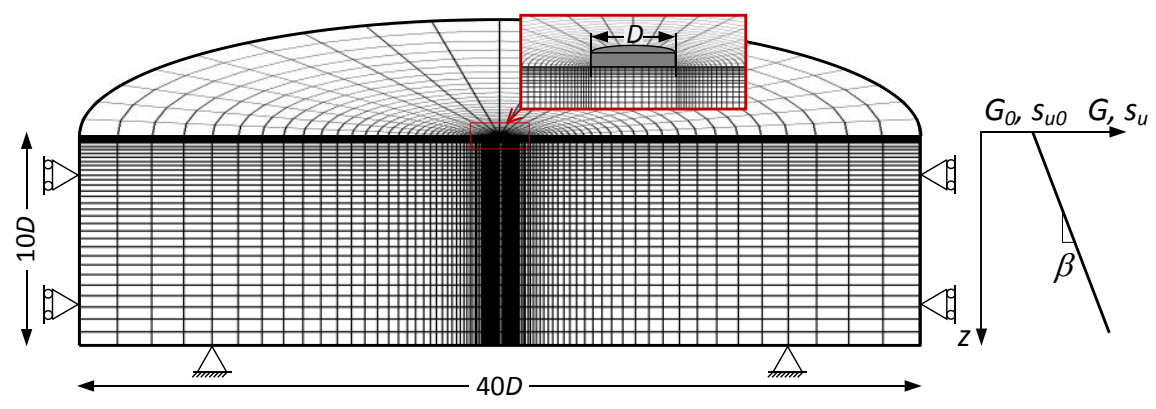

Fig. 2. Finite difference grid of the $F L A C^{3 D}$ model.

The size of the soil grid (radius 20D, depth 10D) was selected in order to simulate a half-space condition of unbounded soil. Previous studies have shown that using dimensions which are just sufficient for predicting the foundation capacity (say, within 1\% accuracy when estimating the vertical bearing capacity) may lead to $10-20 \%$ overprediction of the initial tangential stiffness [22, 23]. Therefore, the accuracy in prediction of the elastic foundation stiffness for homogeneous deposits was used as the criterion for selection of appropriate grid dimensions. Gazetas [24] showed that soil non-homogeneity tends to reduce the " depth of influence" in response to static vertical, horizontal forces and bending moment. This indicates that the grid size used for a homogeneous soil medium should also be valid for non-homogeneous conditions. Using the $\mathrm{FLAC}^{3 \mathrm{D}}$ model shown in Fig. 2 , the predicted ultimate capacities and initial stiffnesses (for a homogeneous soil stratum) for vertical, swaying and rocking motions were within $5 \%$ of the analytical solutions [21, 22].

Both load-control and displacement-control methods were used to study the behaviour of the foundation using $\mathrm{FLAC}^{3 \mathrm{D}}$. In the load-control analysis, the foundation was modelled with zones (Young's modulus $E_{f}=35 \mathrm{GPa}$ and Poisson's ratio $v_{f}=0.2$ ) that were separated from the soil using an interface modelled as a collection of linear spring-slider systems. To simulate a bonded interface, the tensile and shear strengths of the interface elements were assigned high values while the normal and tangential stiffness, $k_{n}$ and $k_{s}$ were calculated using the rule-of-thumb estimate recommended by Itasca Consulting Group[19]:

$$
k_{n}=k_{s}=10 \times \max \left[\frac{(K+4 / 3 G)}{\Delta z_{\min }}\right]
$$

where $K$ and $G$ are the bulk and shear moduli of all zones adjacent to the interface and $\Delta z_{\min }$ is the smallest width of an adjoining zone in the normal direction. In this way, the relative displacement between the foundation and soil is mainly controlled by the stiffness and strength of the saturated clay. The load-control technique was used in the unidirectional loading tests to determine the loaddisplacement responses of the foundation under pure vertical or horizontal forces as well as bending moment. 
Two sets of displacement-control tests were carried out in this study. In the first set of tests (referred to as DC-1), the foundation was not physically modelled but represented by the area of its base on the surface of the soil. Within the area, controlled displacements were applied to the nodes that were constrained in the horizontal direction to simulate the rigidity and roughness of the foundation. The first set of displacement-control tests were used for identifying the loaddisplacement relations of the foundation in response to pure external loads and moments (results shown in Fig. 4). The second set of displacement-control tests (referred to as DC-2) utilized the same foundation models used in the load-control tests (identical foundation and interface properties). In addition, in each of these tests, a stiff column was mounted on the foundation to simulate a relatively rigid superstructure, with a controlled horizontal displacement applied to the column at the effective height in accordance with a prescribed $M /(H D)$ ratio (Fig. 3). When loaded to failure through a specific load path, the corresponding failure point of the foundation was found. The failure envelope, defined as the closed surface where all possible failure points reside in the $V: H: M$ space, was then derived from a number of tests conducted with different load paths.

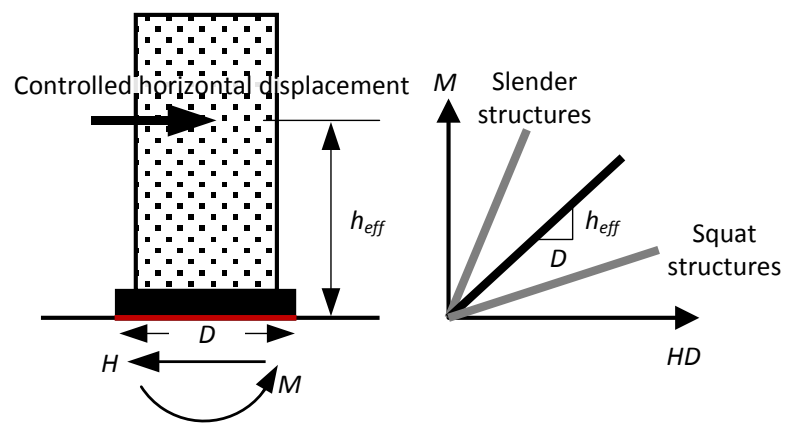

Fig. 3. Idealized seismic load paths generated by the application of a controlled horizontal displacement to a specific height of the superstructure.

\subsection{Load-Displacement Response}

The tests to investigate the load-displacement characteristics of the foundation were first performed in a load-control fashion under a unidirectional loading condition. The controlled loading was specified by imposing nodal forces to the foundation at appropriate increments. The global forces $V$, $H$ and moment $M$ can be calculated either by enforcing the equilibrium condition to the foundation or by evaluating the integral of the stresses over the area of the interface. Both approaches have been adopted to confirm consistency of results. A maximum allowable unbalanced force ratio of $1 \times 10^{-6}$ was used as the convergence criterion.

To confirm the results of the load-control tests, an initial set of displacement-control tests was conducted in FLAC ${ }^{3 \mathrm{D}}$ by applying controlled uniform velocity (in order to simulate a rigid foundation) to the "foundation nodes" on the surface of the soil half-space. The global forces were calculated as the integral of the accumulated unbalanced nodal forces, whereas the global displacements were evaluated as the integral of the velocity over the steps. A velocity of $2 \times 10^{-7} \mathrm{~m} / \mathrm{step}$ was used to obtain the vertical and horizontal response of the foundation while an angular velocity of $5 \times 10^{-8}$ $\mathrm{rad} / \mathrm{step}$ was used for analysing the rocking response. An advantage of using the displacementcontrol analysis is that there is no need to model the stiffness of the foundation or the interface, therefore the effect of these elements is not included in the DC-1 model results. 
A good agreement between results from force- and displacement-control tests was found, as shown in Fig. 4 which depicts the dimensionless load-displacement responses of the foundation on a homogeneous soil $(\lambda=0)$ under unidirectional loading. The dimensionless horizontal and vertical forces and the overturning moment are defined as $H /\left(A s_{u 0}\right), V /\left(A s_{u 0}\right)$ and $M /\left(A D s_{u 0}\right)$, respectively. Numerical results shown in Fig. 4 give a good match to the exact analytical values of 1.0, 6.05 and 0.67 [21]. It was concluded that the parameters adopted for the foundation and interface stiffness in the load-control tests were appropriate given the good agreement obtained with the DC-1 results.

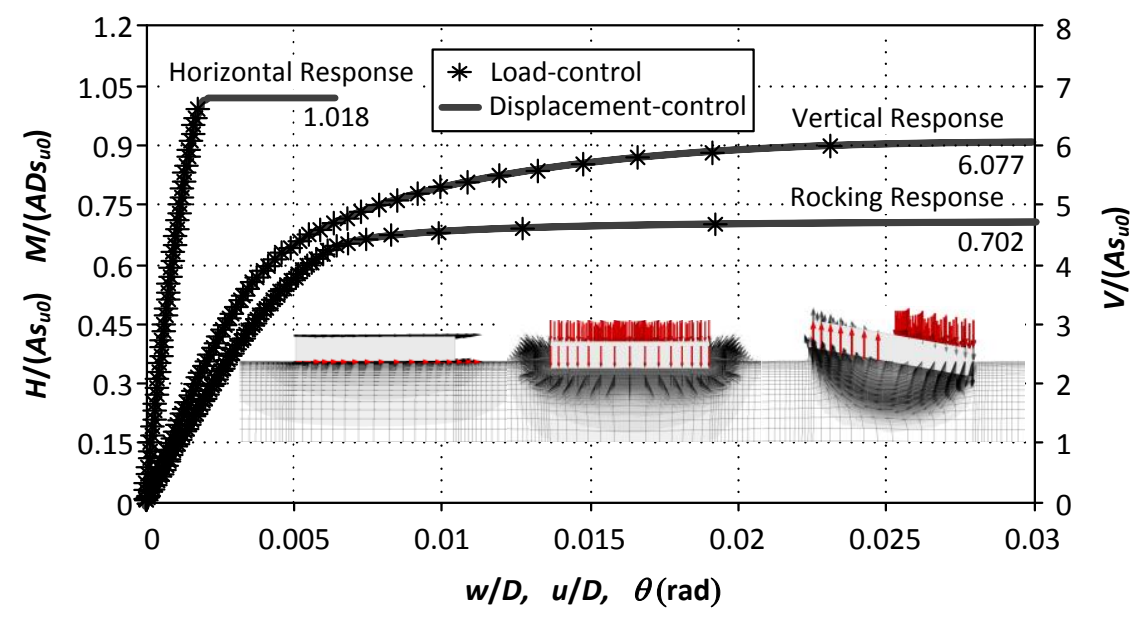

Fig. 4. Force-displacement responses of a circular surface foundation on a homogeneous soil halfspace under unidirectional loading in load-control and displacement-control (DC-1) tests.

In a second set of displacement-control tests, the path-dependent load-displacement response was investigated through a series of push-over analyses. Letting $V_{u}, H_{u}$ and $M_{u}$ denote the ultimate foundation capacities due to the pure forces and moments in the load-control tests, the factor of safety against vertical bearing capacity failure $F S v$ is defined as the ratio $V_{u} / V$. In these displacementcontrol tests, a vertical downward velocity $\left(1 \times 10^{-7} \mathrm{~m} / \mathrm{step}\right)$ was firstly applied to the nodes on the surface of the foundation and superstructure until the sum of the measured accumulated unbalanced nodal forces was, within $0.1 \%$ accuracy, equal to the desired vertical load level $V$ for a given value of FSv. Secondly, these unbalanced nodal forces were applied to the corresponding foundation nodes, whose degrees of freedom were then set free to achieve the load and stress state for the given FSV. This was followed by the application of a horizontal displacement (at $2 \times 10^{-8}$ $\mathrm{m} / \mathrm{step}$ ) to the height of the superstructure prescribed for a given moment-to-shear ratio $M /(H D)$.

Fig. 5 presents the push-over test results corresponding to different values of FSV and moment-toshear ratios for a homogeneous soil deposit. The tested range of moment-to-shear ratio $M /(H D)$ from 0.5 to 1.25 represents a typical building structure having a small-to-moderate slenderness ratio. It can be observed that the initial stiffness of the foundation (after applying the vertical load) reduces with decreasing FSv, especially for the rocking response (graphs a); whereas it is almost unaffected by the load ratio (graphs b). Results for nonhomogeneous soil deposits are very similar to those presented in Fig. 5 despite the fact that the variation of initial stiffness with FSv is less significant when increasing the soil heterogeneity (i.e. increasing the $\lambda$ value). The reduction of stiffness was the consequence of soil yielding during the first loading phase, where the penetration of the foundation into the underlying soil induced plasticity. 

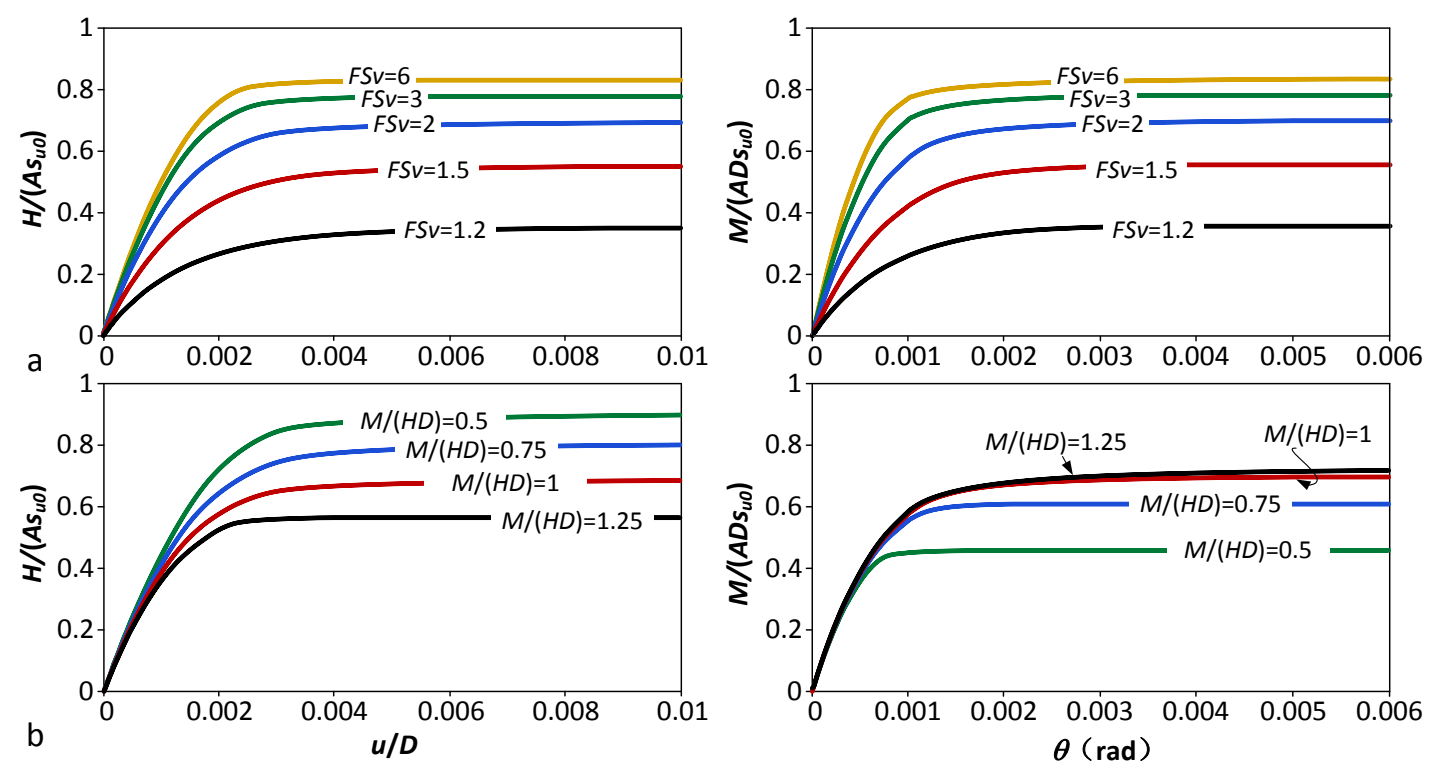

Fig. 5. Push-over curves for the foundation on a homogeneous soil $(\lambda=0)$ for (a) different FSv with $M /(H D)=1$, and (b) different $M /(H D)$ with $F S v=2$.

\subsection{V:H:M Failure Envelope}

Although the push-over tests enable the path-dependent load-displacement curve and the corresponding failure point to be obtained simultaneously, it is not a convenient way to develop the whole failure envelope representing the ultimate state of the foundation, simply due to the fact that each test can only determine a single point on the failure surface. Alternatively, a "swipe" test may be introduced, where a single displacement-control test can yield a failure curve across the 3D failure surface. The "side-swipe" test was proposed by Tan [25] and has been adopted by various researchers to identify the failure envelope (e.g. $[26,27])$. This type of test is performed in the displacement space where the foundation is brought to failure by increasing the displacement in one direction $u_{1}$, followed by imposing displacement in the second direction $u_{2}$ while maintaining $u_{1}$ (i.e. $\Delta u_{1}=0$ ). During the second loading phase, the load in the first direction decreases with a reduction of the corresponding elastic displacement $u_{1 e}$. This is accompanied by an increase in the plastic displacement $u_{1 p}$ to maintain the condition that $\Delta u_{1}=0$. As a result, the load path is believed to track close to the failure locus in the load space.

An example of the "swipe" test results is shown in Fig. 6 (a) where the Finite-Element (FE) results of Gourvenec [21] are used as a comparison for the obtained failure envelope. This example corresponds to tests where the foundation was rotated to failure and then driven horizontally along the soil surface at a fixed angle of rotation. The failure points obtained from the push-over analyses are also plotted to compare with the "swipe" test results. The agreement between the three sets of results is fairly good. In addition, Fig. 6 (b) illustrates a 3D representation of the failure surface for the homogeneous soil condition, along with two of the preferred seismic load paths, in the normalized load space. It should be mentioned that under a fixed load path of $M /(H D)=1.25$, the foundation resting on a uniform soil deposit showed a reversed (backward) translation at failure for $F S v=3$ and 6; while for the non-homogeneous soil conditions, the foundation kept moving forward at failure. This is a result of the associated flow rule on the asymmetric $M-H$ failure envelope. 


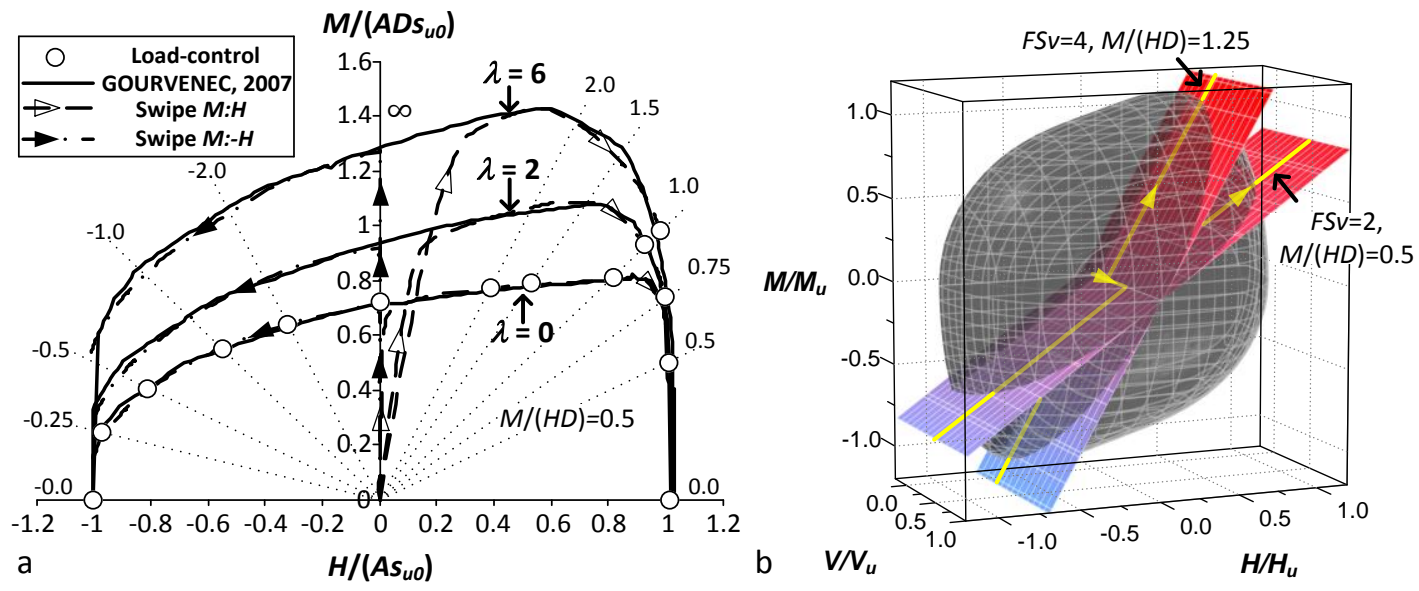

Fig. 6. (a) Comparison of test results for the $M-H$ interaction diagram of the failure envelope for $F S v=\infty$ and (b) 3D failure surface of the foundation $(\lambda=0)$ and desirable load paths in the normalized $V: H: M$ space.

\section{Simplified NSR Model}

As described in section 2, the NSR model consists of spring-dashpot systems capable of capturing the nonlinear sway and rocking responses of a shallow foundation. This section presents the mathematical formulations for characterizing the behaviour of each model component as well as the model calibration procedures.

\subsection{Model Description}

After reaching static equilibrium under vertical self-weight loading, the behaviour of the soilfoundation system in response to a small displacement is dictated by the initial stiffness $k_{i n}$. Fig. 5 showed that this stiffness drops as the safety factor FSV decreases and that there is no significant degradation of the initial stiffness at low displacement levels. It is therefore assumed that the smalldisplacement response of the foundation, corresponding to either the sway or rocking degree of freedom, can be described using a linear relationship given by:

$$
F=k_{i n} u_{e}
$$

where the global force $F$ is calculated as the product of the initial stiffness $k_{i n}$ and the displacement $u_{e}$. The range of the linear portion of the load-displacement curve is defined by:

$$
F_{i n}=C_{r} F_{c}
$$

where $F_{\text {in }}$ is the global force when first entering the plastic cycle and $C_{r}$ is the ratio of $F_{\text {in }}$ to the capacity $F_{c}$.

The plastic component of the $p-y$ springs developed by Boulanger et al. [28] is adopted here to simulate the large-displacement response. The $p-y$ springs were initially used in soil-pile interaction analyses to model the response of laterally loaded piles. In the NSR model, the plastic spring is assumed to be rigid when $|F|<F_{\text {in }}$ in virgin loading. This rigid range of $2 F_{\text {in }}$ translates with plastic loading during which the nonlinear monotonic force-displacement curve of the plastic spring follows the relation: 


$$
F=F_{c}-\left(F_{c}-F_{0}\right)\left[\frac{c u_{50}}{c u_{50}+\left|u_{p}-u_{p 0}\right|}\right]^{n}
$$

where $u_{p}$ is plastic displacement component; $F_{0}$ and $u_{p o}$ are, respectively, the global force and plastic displacement at the start of the current plastic loading cycle; $u_{50}$ is the total displacement $\left(u_{e}+u_{p}\right)$ at which $50 \%$ of $F_{c}$ is mobilized in monotonic loading; and $c, n$ are constants that control the shape of the overall load-displacement curve of the foundation.

The radiation damping is assumed to be of viscous type and modelled through a dashpot placed in parallel with the linear spring, while the hysteretic damping of the foundation is naturally accounted for by the nonlinearity embedded in the load-displacement behaviour during the loading-unloadingreloading cycles. Existing analytical expressions allow the radiation damping coefficients to be calculated from the stiffness $k_{\text {in }}$ (e.g. [29]). It should be noted that during strong shaking events, the radiation damping becomes negligibly small compared to hysteretic damping.

The global load-displacement behaviour of the foundation, shown in Fig. 7, is implemented in OpenSees [30] for each of the swaying $\left(F, k\right.$ replaced by $H, k_{h}$, respectively) and rocking $(F, u, k$ replaced by $M, \theta, k_{r}$, respectively) responses. Detailed descriptions of the model components are provided by Boulanger et al. [28, 31].

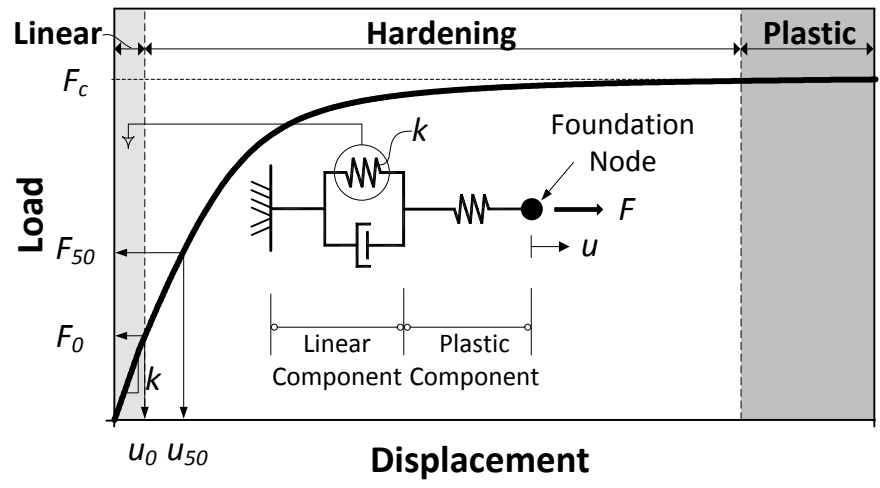

Fig. 7. Nonlinear load-displacement response of a foundation modelled by the equivalent springdashpot system.

\subsection{Model Calibration}

\subsubsection{Initial stiffness}

This section presents a description of the model calibration procedure that was performed utilizing existing analytical and empirical expressions, as well as numerical results from the static $\mathrm{FLAC}^{3 \mathrm{D}}$ simulations. In practice, it is often required that a safety factor FSV greater than 2 should be used to avoid excessive settlement; hence, results with FSV less than 2 were not considered in the calibration. In addition, data for $M /(H D)=1.25$ with $\lambda=0$ and $F S v>2$ were also not considered in developing the model because of the backward foundation translation explained in section 3.3.

The initial swaying or rocking stiffness of the foundation corresponds to the condition where the vertical load $V$ is fully developed during the construction and service period before any shaking takes 
place. It is convenient to express the initial stiffness $k_{i n}$ as a fraction of its purely elastic counterpart $k$ ( $k_{h}$ and $k_{r}$ correspond to swaying and rocking degrees of freedom, respectively) as:

$$
k_{\text {in }}=\alpha(F S v, \lambda) k
$$

where $\alpha$ ( $\alpha_{h}$ and $\alpha_{r}$ correspond to swaying and rocking motions, respectively) is a stiffness loss factor which is a function of FSV and $\lambda$. The variation of $\alpha$ with FSV for different soil heterogeneity is depicted in Fig. 8 for the swaying and rocking motions. The data shows that the rocking stiffness drops more significantly than the swaying stiffness when reducing the factor of safety FSV.
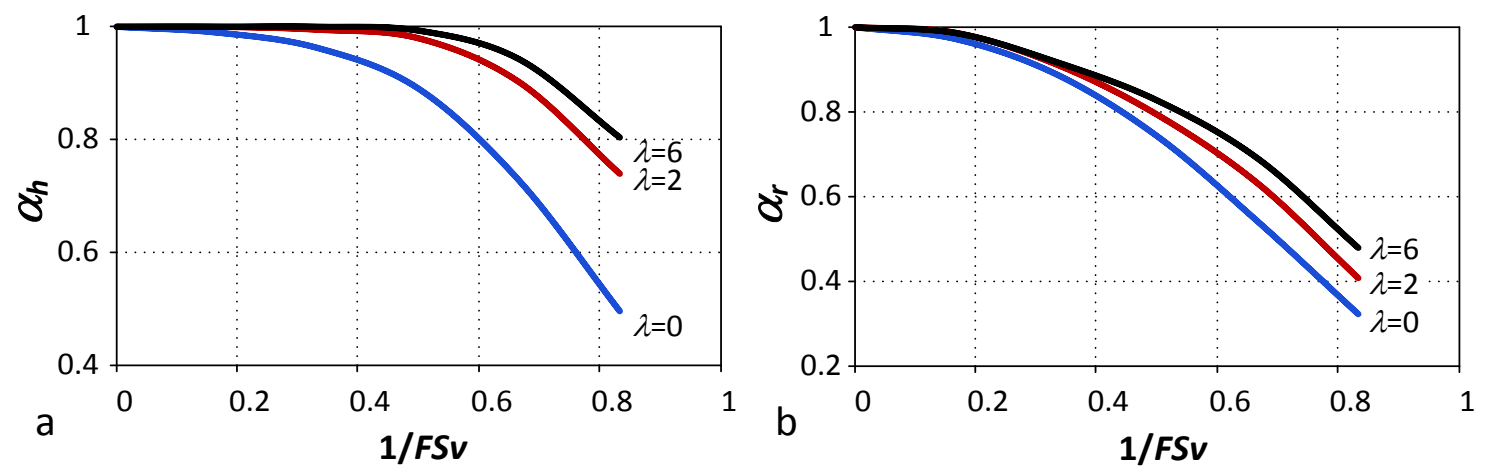

Fig. 8. Variation of initial foundation stiffness with vertical load safety factor for (a) swaying and (b) rocking responses.

In general, the swaying and rocking responses of a rough surface foundation bonded to the surface of a linear elastic half-space are coupled, which can be expressed in the following matrix form:

$$
\left[\begin{array}{l}
H \\
M
\end{array}\right]=\left[\begin{array}{cc}
k_{h} & k_{h r} \\
k_{r h} & k_{r}
\end{array}\right]\left[\begin{array}{l}
u \\
\theta
\end{array}\right]
$$

where $k_{h r}$ and $k_{r h}$ account for the cross-coupling between the swaying and rocking stiffness. Bell [22] demonstrated that this coupling effect is more pronounced for lower values of Poisson's ratio $v$ (e.g. $v<0.4)$. In the case of undrained conditions, where the saturated soil is idealized as being incompressible $(v=0.5), k_{h r}$ and $k_{r h}$ are zero. Therefore, in this study, the linear swaying and rocking responses are assumed to be independent of each other so that $k_{h r}=k_{r h}=0$ and $k_{h}, k_{r}$ can be calculated using the following expressions:

$$
\begin{aligned}
& k_{h}=\chi_{h}(\lambda) \frac{4 G_{0} D}{2-v} \\
& k_{r}=\chi_{r}(\lambda) \frac{G_{0} D^{3}}{3(1-v)}
\end{aligned}
$$

where $G_{0}$ is the small strain shear modulus of the soil at ground level and $\chi$ is a dimensionless influence factor that takes into account the effect of soil non-homogeneity. Note that for a uniform soil condition (i.e. $\lambda=0), k_{h}=4 G_{0} D /(2-v)$ and $k_{r}=G_{0} D^{3} /[3(1-v)]$ are analytical expressions for swaying and rocking stiffnesses given by Poulos and Davis [32]. A number of investigations have been carried out to study $k_{h}$ and $k_{r}$ for surface foundations on non-homogeneous soil deposits (e.g. [24, 33-37]). 
Some of these studies found that the swaying stiffness is more sensitive to the rate of nonhomogeneity $\lambda$, whereas the rocking stiffness is less affected $[24,35,36]$. Based on these studies, Gazetas [29] proposed the following empirical expressions for estimating $\chi(\lambda)$, where the subscripts $h$ and $r$ refer to swaying and rocking, respectively:

$$
\chi_{h}(\lambda) \approx 1+0.22 \lambda, \quad \chi_{r}(\lambda) \approx 1+0.15 \lambda
$$

It should be mentioned that most of the stated studies on which Eq. (11) is based, assumed a drained soil condition by using a constant Poisson's ratio of $v=0.25$ or 0.33 . Results from this study, however, show that under an undrained condition $(v \rightarrow 0.5)$, the rocking stiffness experiences a larger increase than the swaying stiffness when soil non-homogeneity increases. Fig. 9 compares the variation of the influence factor $\chi$ predicted in this study with those estimated using Eq. (11) for two values of Poisson's ratio $v=0.25$ and 0.49 .

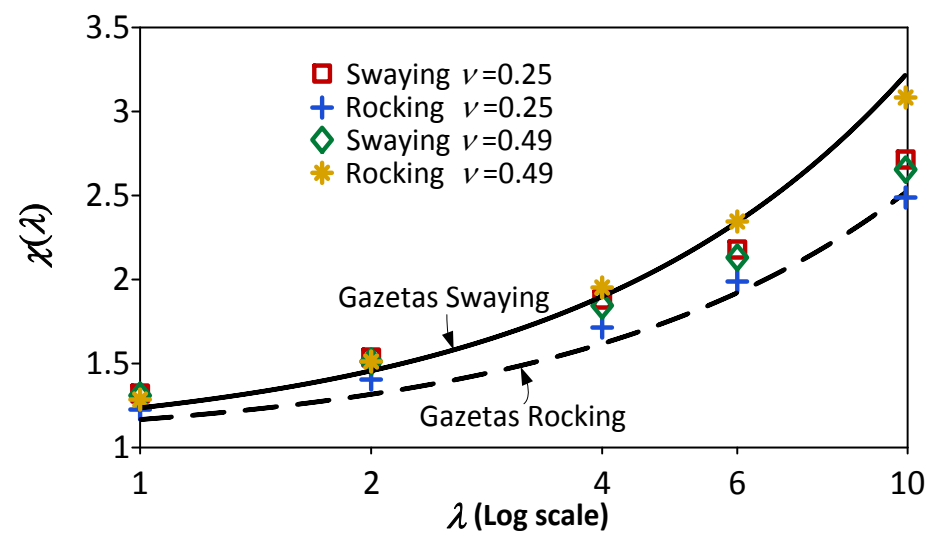

Fig. 9. Effect of soil non-homogeneity and Poisson's ratio on elastic swaying and rocking stiffnesses.

The comparison demonstrates that for a wide range of heterogeneity $(1<\lambda<10)$, the variation of the swaying stiffness is practically independent of Poisson's ratio $v$, while the rocking stiffness increases more rapidly in an undrained condition than in a drained condition. Similarly, Carrier and Christian [38] observed that for a rigid circular surface foundation lying on a generalized "Gibson" soil halfspace, the vertical stiffness increases much faster with heterogeneity for higher values of Poisson's ratio. It can be concluded that the variation of foundation stiffness with soil heterogeneity for the vertical and rocking motions is much more sensitive to Poisson's ratio than for the swaying response. Fig. 9 also illustrates that Gazetas's expressions for $\chi(\lambda)$ work reasonably well for estimating the swaying stiffness for $\lambda \leq 4$, while underestimating the undrained rocking stiffness. It should be noted that in application of the proposed model to dynamic loading, the frequency dependence of the stiffness and radiation damping was ignored since the emphasis was given to the post-yield response of the foundation where large displacements were expected to occur. In the developed NSR model, the initial foundation stiffness was evaluated by applying the values of $\alpha$ and $\chi$ obtained from the $\mathrm{FLAC}^{3 \mathrm{D}}$ analyses and illustrated in Figs. 8 and 9, respectively, to the previously stated analytical solutions for $k_{h}$ and $k_{r}$ for uniform soil conditions.

\subsubsection{Coupled bearing capacity}

The coupled shear and moment capacities $H_{c}$ and $M_{c}$ correspond to the intersection between the load path and the failure envelope in the load space. Gourvenec [21] proposed that the normalized 
moment capacity $M_{c} / M_{u}$ could be approximated as cubic or quartic polynomials with respect to 1/FSv:

$$
\begin{cases}\frac{M_{c}}{M_{u}}=c_{1}\left(\frac{1}{F S v}\right)^{3}+c_{2}\left(\frac{1}{F S v}\right)^{2}+c_{3}\left(\frac{1}{F S v}\right)+c_{4} & \left(M_{c} \cdot H_{c}>0\right) \\ \frac{M_{c}}{M_{u}}=c_{1}\left(\frac{1}{F S v}\right)^{4}+c_{2}\left(\frac{1}{F S v}\right)^{3}+c_{3}\left(\frac{1}{F S v}\right)^{2}+c_{4}\left(\frac{1}{F S v}\right)+c_{5} & \left(M_{c} \cdot H_{c}<0\right)\end{cases}
$$

where $c_{1}$ to $c_{5}$ are polynomial coefficients given in [21] for a number of discrete values of normalized moment-to-shear ratios $M_{d} /\left(N_{c M} H_{c} D\right)$ and non-homogeneity index $\lambda . N_{c M}$ is the ultimate moment capacity coefficient defined as the ratio of $M_{u} /\left(A D s_{u 0}\right)$. Since the base shear and moment induced by the horizontal ground accelerations always act in the same direction (see Fig. 1 for sign convention), only the cubic polynomial expression in Eq. (12) is necessary for calculating the moment capacity. In preliminary seismic design, it is usually assumed that the dynamic behaviour of a fixed-base MultiDegree-of-Freedom (MDOF) structure is dominated by its fundamental mode of vibration such that it can be treated as a SDOF system; the following relation can thus be obtained:

$$
\frac{M_{c}}{H_{c}}=h_{e f f}=\frac{\sum_{i=1}^{N} m_{i} \varphi_{i 1} h_{i}}{\sum_{i=1}^{N} m_{i} \varphi_{i 1}}
$$

where $N$ is the number of storeys, $m_{i}, h_{i}$ and $\varphi_{i 1}$ are, respectively, mass, height (measured from ground floor) and amplitude at the $i^{\text {th }}$ storey corresponding to the first mode of vibration of a MDOF structure. More accurate values of $h_{\text {eff }}$ for flexible-base buildings can be calculated by taking into account the linear foundation springs described in section 4.2.1 through a standard modal analysis in structural dynamics.

\subsubsection{Hard-coded shape parameters}

The shape of the nonlinear backbone curve for shallow foundations is mainly controlled by the parameters $C_{r}, c$ and $n$ (from Eq. 6), the initial foundation stiffness, ultimate capacity, and the displacement corresponding to $50 \%$ of the capacity $\left(u_{50}\right)$. Although $C_{r}$ specifies the range of the linear segment of a backbone curve, the push-over curves in the numerical tests do not possess a strictly linear portion. An example (for the uniform soil condition) is shown in Fig. 10, where secant foundation stiffness $k_{s e c}$, normalized by its initial value, is plotted against the mobilized strength $(H$ and $M)$ normalized by corresponding ultimate values $\left(H_{c}\right.$ and $\left.M_{c}\right)$.
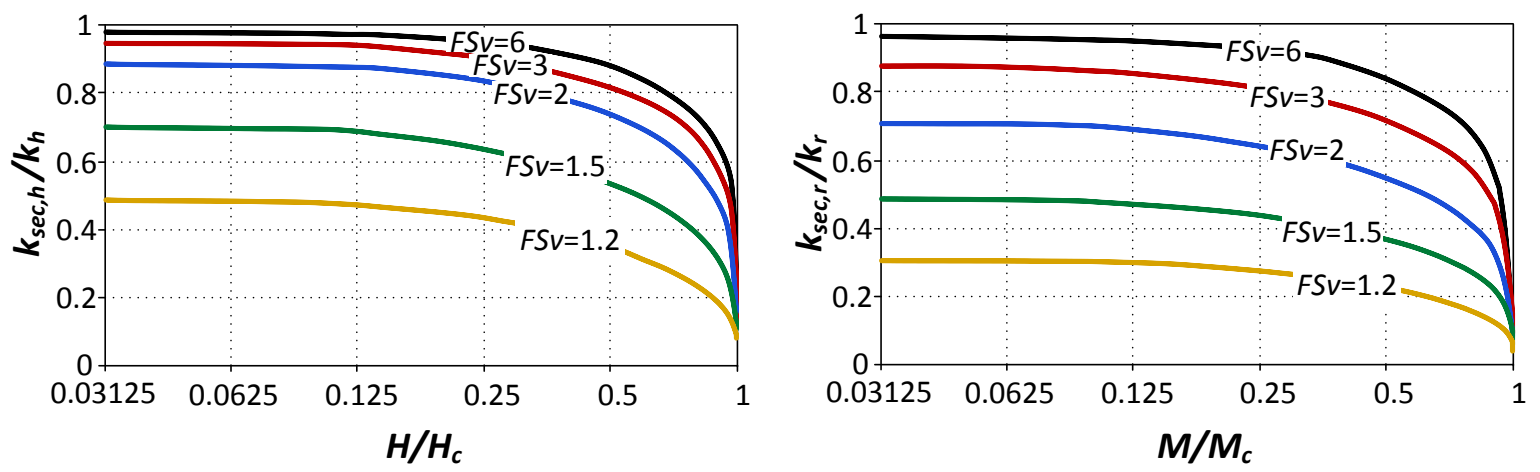
Fig. 10. Variation of secant foundation stiffness with mobilized foundation load level.

As seen in Fig. 10, the foundation stiffness gradually reduces with increasing load level. This reduction, however, is not significant when the horizontal load and moment are (approximately) lower than 0.125 of their ultimate values (which was also the case for $\lambda=2$ and 6 ). Therefore, this value was used as the linear range $C_{r}$ in the current model. The reference displacement $u_{50}$ can be related to a dimensionless parameter $c_{50}$ defined as [16]:

$$
c_{50}=\frac{k_{i n} u_{50}}{F_{c}}
$$

$c_{50}$ was calculated for each of the FD push-over analyses according to this Eq.. It was found in this study that regardless of $F S v, M /(H D)$ and $\lambda$, the calculated $c_{50}$ values were fairly close to each other. For example, the mean and standard deviation of $c_{50}$ for the swaying response were 0.562 and 0.027 ; for the rocking response they were 0.561 and 0.042 . Therefore, the mean values of $c_{50}$ were hard coded into the model.

By normalizing the load and displacement with respect to $F_{c}$ and $u_{50}$, respectively, the shape of the backbone curves tends to be unique, as shown in Fig. 11. Calibration of $c$ and $n$ were then carried out through identifying the "best fit" values, presented in Table 1, which minimized the "error" between the analytical (Eqs. (4) and (6)) and the numerical backbones. The square root of the sum of the squared force residuals over all displacement points was used to measure the error [16]. The analytical backbone curves for soil heterogeneities $\lambda=0,2$ and 6 were found to be similar to each other, as illustrated in Fig. 12. Based on the results illustrated in Figs. 11 and 12, for practical purposes, a unique normalized backbone curve could be adopted for each of the swaying and rocking motions. Note that "unique" in this context refers to independence of factor of safety FSv, moment-to-shear ratio $M /(H D)$, and soil heterogeneity $\lambda$.

Table 1. Shape parameters for the simplified NSR model.

\begin{tabular}{ccccccc}
\hline & \multicolumn{3}{c}{ Swaying Response } & \multicolumn{3}{c}{ Rocking Response } \\
\hline & $\lambda=0$ & $\lambda=2$ & $\lambda=6$ & $\lambda=0$ & $\lambda=2$ & $\lambda=6$ \\
\hline$c$ & 0.49 & 0.16 & 0.10 & 0.48 & 0.42 & 0.46 \\
$n$ & 1.94 & 0.91 & 0.63 & 1.72 & 2.11 & 2.08 \\
\hline
\end{tabular}



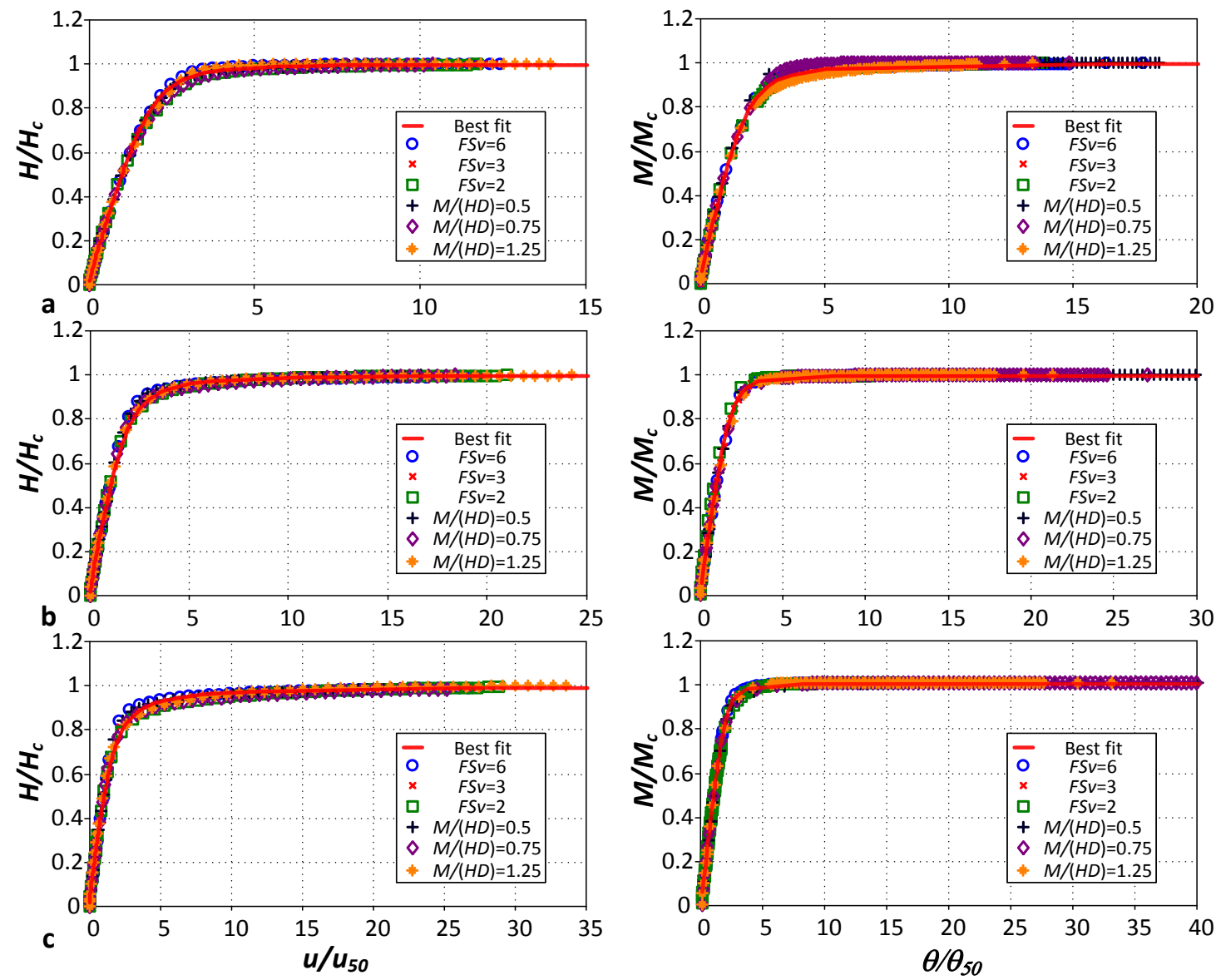

Fig. 11. Normalized load-displacement curves showing a unique shape compared with the analytical fit for three values of factor of safety $F S v=2,3,6$ (under a constant $M /(H D)=1$ ) and four moment-toshear ratios $M /(H D)=0.5,0.75,1,1.25$ (at a constant $F S v=2$ ), corresponding to soil heterogeneities (a) $\lambda=0$, (b) $\lambda=2$ and (c) $\lambda=6$;
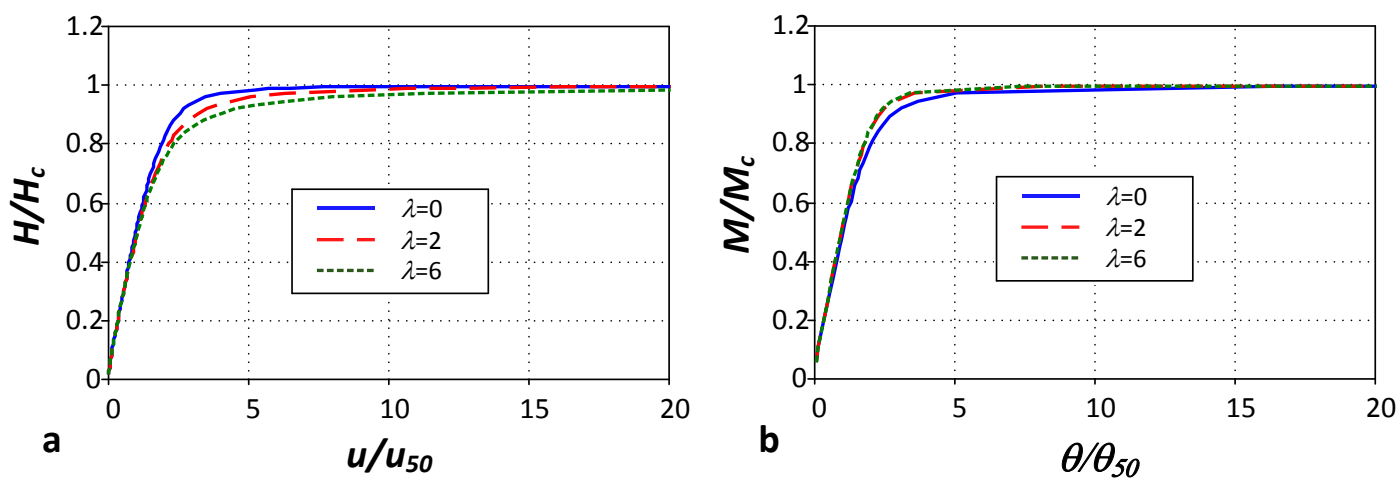

Fig. 12. Normalized load-displacement curves for (a) foundation swaying response and (b) foundation rocking response with a similar shape for various degrees of soil heterogeneity.

\section{NSR Model Validation}

In this section, results from nonlinear dynamic analyses performed using both rigorous FAC $^{3 D}$ simulations and the simplified NSR model are compared to demonstrate the effectiveness and efficiency of the simplified model. Note that the $F L A C^{3 D}$ model used in this section differs from the static analysis model described previously. 


\subsection{Soil-Structure Model in Dynamic Analysis}

The finite-difference grid of the soil-structure system used in the dynamic analysis is shown in Fig. 13, where the superstructure is represented by a cylindrical aluminium column. A fine mesh was used close to the foundation and a coarser mesh was used near the sides and base of the soil domain. To avoid numerical distortion of the propagating wave, the maximum allowable mesh size was controlled within one-tenth to one-eighth of the wavelength associated with the highest frequency component of the input wave [39].

In the dynamic analysis, the half-space condition of the unbounded soil was satisfied by applying appropriate boundary conditions. "Free-field" boundaries were specified along the vertical sides of the soil grid to reproduce motions at infinity, whereas "quiet" boundaries were imposed in between the "free-field" and soil side boundaries, as well as at the bottom, to reduce the reflection of outward propagating waves back into the model. The "quiet" boundaries are modelled using dashpots that are placed independently in the normal and tangential directions with respect to the soil boundaries. During the dynamic analysis in FLAC ${ }^{3 \mathrm{D}}$, the motion within the model and the "freefield" motion (in the absence of the structure and foundation) are calculated simultaneously, and the unbalanced forces at the "free-field" grid-points are then applied to the soil-structure system through the corresponding grid-points at the soil boundaries [13].
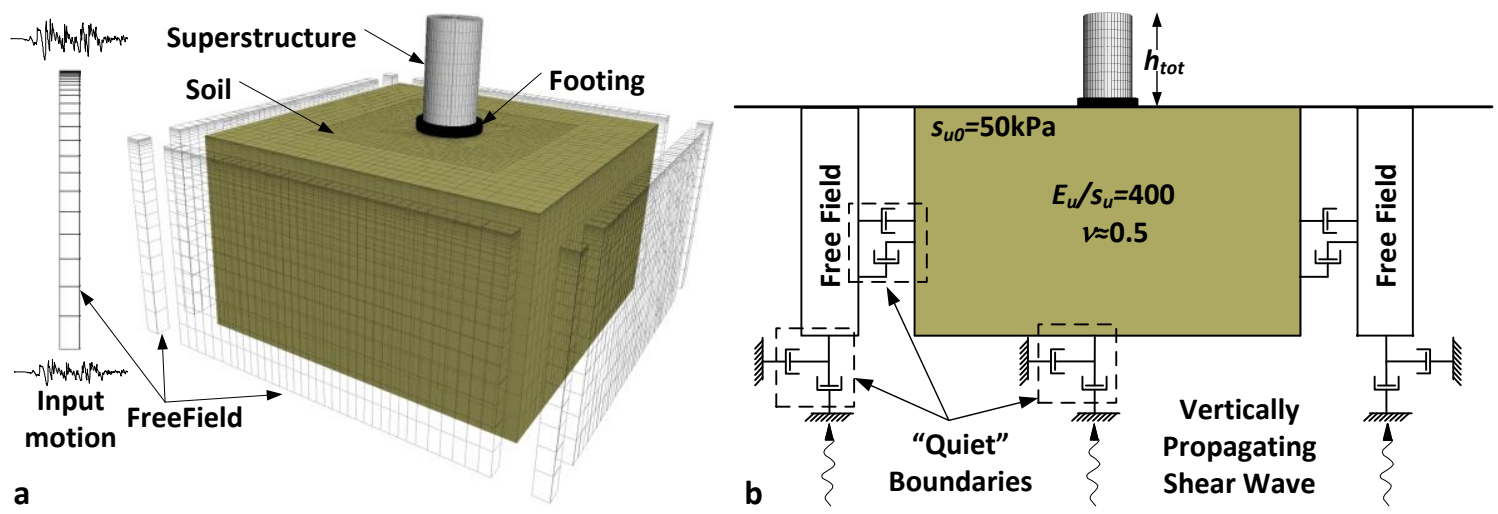

Fig. 13. (a) Finite-difference grid and, (b) boundary conditions of the soil-structure model used in the nonlinear dynamic analysis.

It should be mentioned that the linear elastic perfectly plastic soil model has a limitation in modelling dynamic soil behaviour, especially at small to medium strain levels. This class of model may be extended to account for hardening effects that are able to describe pre-failure nonlinearity in addition to the accumulation of irrecoverable strains [40]. While the model cannot completely capture the hysteretic behaviour in response to the cyclic loading, FLAC ${ }^{3 D}$ provides an optional "hysteretic damping" model utilizing a variety of stress-strain backbone curves and Masing rules [41] to simulate the material damping at small strains. However, the use of this damping should be treated with care and justified when combined with a yield model. One issue with the "hysteretic damping" model is that the stiffness degradation causes large strains, but the "hysteretic damping" is not intended to simulate yielding at this strain level. Moreover, the reduction of stiffness with increasing strain may lead to unrealistic response as it modifies the dynamic properties of the system. 
It is suggested by Itasca Consulting Group [19] that a trial simulation should be run with an assumed linear material model to identify the large strain regions where the "hysteretic damping" must be excluded. In other words, the use of the "hysteretic damping" is subject to a case-by-case variability, which is a function of the stiffness, strength of the material, model geometry and applied load magnitude. Considering the generality of the proposed model, the "hysteretic damping" model was therefore not adopted in this study. Instead, five percent Rayleigh damping was applied to the FD model. As the focus of this study is on the mobilization of foundation bearing capacity during strong shaking, the Tresca plasticity model is sufficient to model the large strain behaviour where a considerable amount of energy dissipation would occur during plastic flow.

The simplified NSR model, as illustrated in Fig. 14, was constructed in the OpenSees [30] platform. The lateral stiffness of the superstructure was modelled by an elastic beam-column element connecting the masses of the foundation and the structure at both ends, whereas the global forcedisplacement response of the foundation was simulated by the uniaxial material developed in section 4 for both swaying and rocking motions. The NSR model requires an input of effective height for the structure, which was calculated based on a trapezoidal horizontal acceleration distribution that is made up of a uniform and a triangular pattern (Fig. 14). In order to give equal importance to each of the swaying and rocking responses, the rectangular and triangular patterns were devised such that each imparted the same lateral resultant force magnitude to the superstructure. The horizontal acceleration pattern illustrated in Fig. 14 results in a value of $h_{\text {eff }}=0.58 h_{\text {tot }}$.

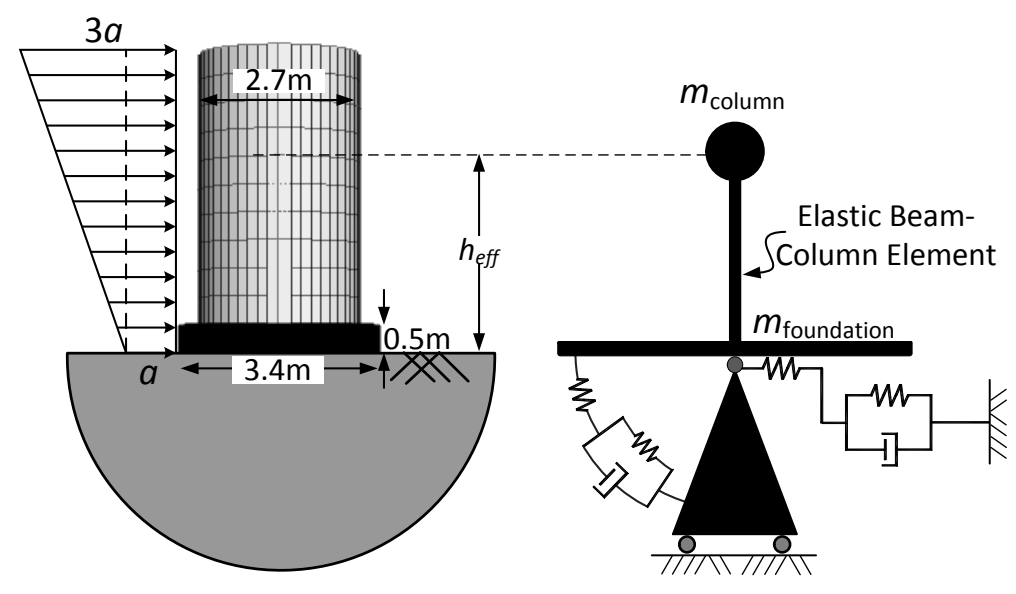

Fig. 14. Simplified NSR model used in the dynamic analysis.

\subsection{Methods and Results}

The analysis in FLAC ${ }^{3 D}$ involved the following three steps: (1) the geostatic state is first achieved by bringing the soil grid to equilibrium under gravity with vertical-roller side-boundaries; (2) the circular foundation and the cylindrical column are then constructed on the soil surface and static equilibrium is solved for a given value of FSv; (3) the roller boundaries are replaced by "free-field" and the "quiet" counterparts followed by dynamic analysis performed by subjecting the grid to the input ground motions applied at the base of the model. Two artificial sinusoidal excitations and a real earthquake acceleration record, shown in Fig. 15, were used as the "free-field" horizontal motions recorded at the ground surface in the absence of the structure. 

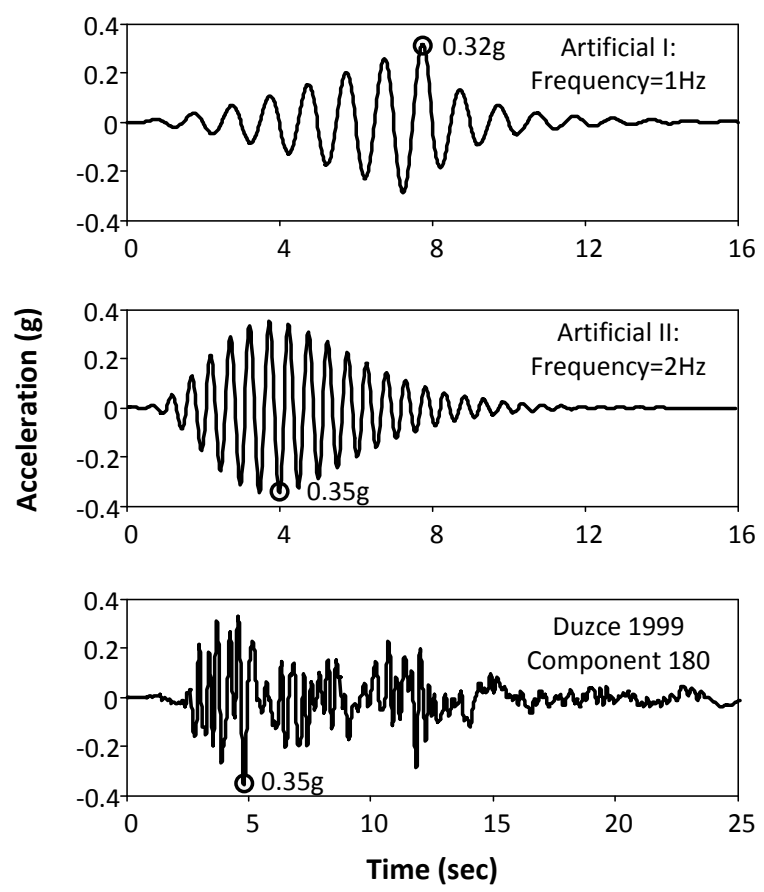

Fig. 15. Artificial and real earthquake ground accelerations utilized as the "free-field" motions recorded at the ground surface.

It should be noted that these "design" acceleration records cannot be used as the input motions for the $\mathrm{FLAC}^{3 \mathrm{D}}$ model. Firstly, the input motions at the base of the $\mathrm{FLAC}^{3 \mathrm{D}}$ model should be determined in order that the motions measured at the ground surface in the "free-field" are recovered as the "design" motions. Secondly, the grid-points on the base of the $\mathrm{FLAC}^{3 \mathrm{D}}$ model should be allowed to move according to the pattern of wave motions so that the "quiet" dashpots can calculate the viscous forces. The former corresponds to a deconvolution process whereas the latter requires the acceleration records to be transformed into stress records which can then be applied to the "quiet boundary" at the base. It should also be mentioned that the input motion for a "quiet" boundary refers to the upward propagating motion rather than the apparent (observed) acceleration within the base [42].

In $\mathrm{FLAC}^{3 \mathrm{D}}$, Rayleigh damping is specified at a centre frequency at which mass damping and stiffness damping each supplies half of the total damping force. When subjected to the artificial ground motions, Rayleigh damping of the SSI system was specified at the frequencies of the excitations while for the real earthquake, the centre frequency was set equal to the middle frequency $(1.8 \mathrm{~Hz}$ in this study) between the lowest and highest predominant frequencies, as suggested by Itasca Consulting Group [19]. In this way, the frequency-independent hysteretic damping could be approximated using Rayleigh damping.

The OpenSees analysis using the NSR model can be used to study the inertial soil-structure interaction, which refers to the interaction between the excited structure and the underlying soil. The effects of kinematic interaction (where the foundation input motion differs from the ground motion due to the inability of the rigid foundation to follow the "free-field" motion) were ignored, which is reasonable for shallow foundations subjected to coherent vertically propagating shear waves [43]. In this case, the "design" motions shown in Fig. 15 were directly applied to the model. Despite some small differences between the shapes of the normalized foundation force- 
displacement backbone curve for various soil heterogeneity levels (shown in Fig. 12), the one derived from the homogeneous soil condition was adopted.

Fig. 16 compares the results predicted by both simplified NSR and more rigorous FLAC ${ }^{3 D}$ models, in terms of shear-sliding and moment-rotation relations for the swaying and rocking motions of the foundation, respectively. The analyses were performed by using various combinations of different design parameters consisting of soil non-homogeneity index $\lambda=0,2,6$, safety factor $F S v=2,3,4$, and slenderness ratio $h_{\text {tot }} / R=2,2.5,3$, where $h_{\text {tot }}$ is the total height of the structure. Considering a typical five-bay building with a bay width of 6 meters and a storey height of 3.3 meters, $h_{\text {tot }} / R=2,2.5$ and 3 approximately correspond to 10,13 and 15 storeys, respectively, if the building is assumed to be symmetric.
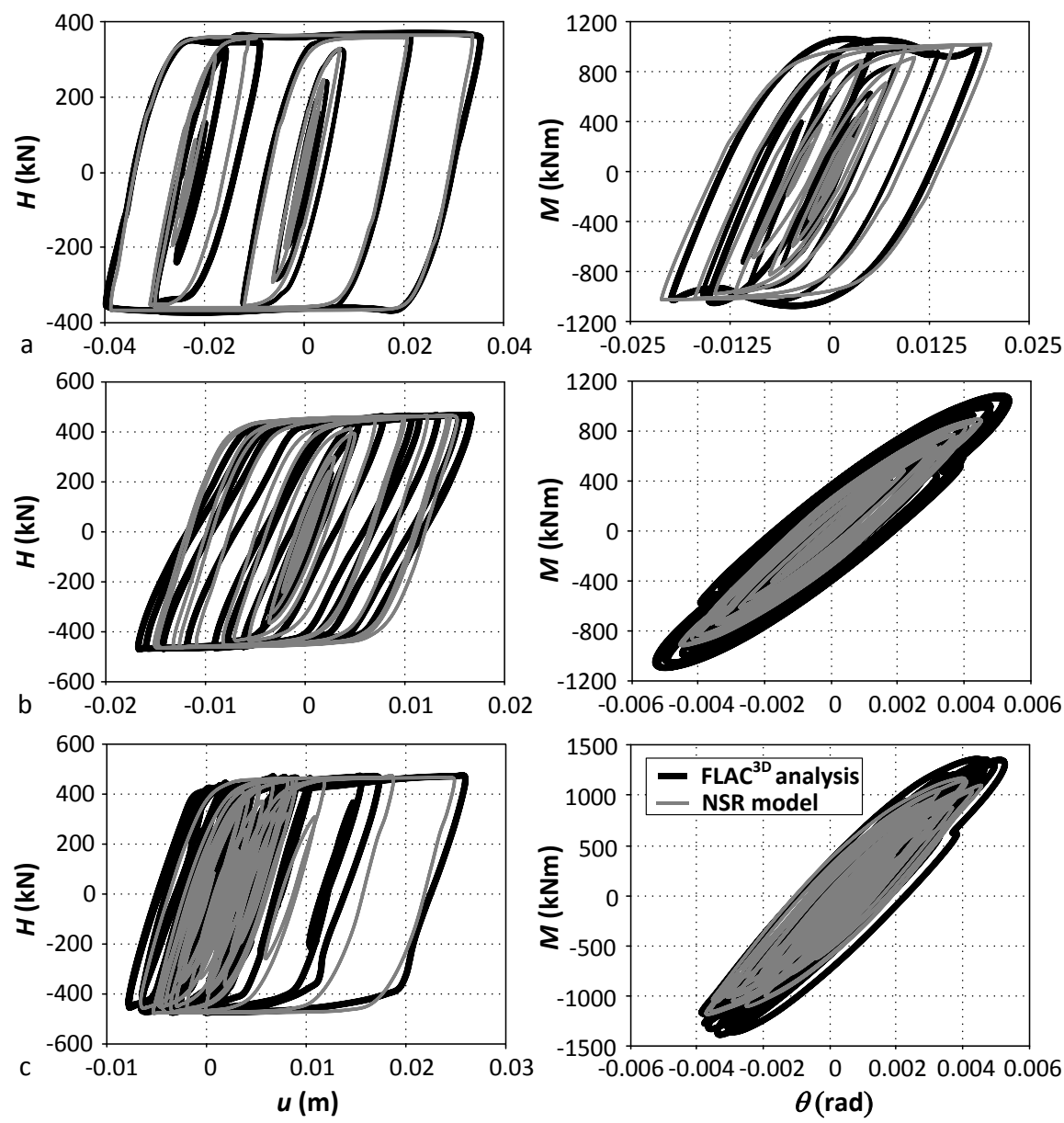

Fig. 16. Comparison of the dynamic load-displacement and moment-rotation foundation responses computed with the FD model (black lines) with those predicted by the NSR model (grey lines) for (a) artificial I ground motion with $F S v=2, h_{\text {tot }} / R=3, \lambda=0$; (b) artificial II ground motion with $F S v=3, h_{\text {tot }} / R=2$, $\lambda=2$; and (c) Duzce 1990 earthquake (component 180) with $F S v=4, h_{\text {tot }} / R=2.5, \lambda=6$.

The comparison shows that, in general, the simplified model is able to reproduce the foundation load-displacement response predicted using the $\mathrm{FLAC}^{3 \mathrm{D}}$ model. Maximum and residual foundation displacements, which are important parameters in a displacement design approach for structures, were also estimated by the simplified model with sufficient accuracy. A significant advantage of the NSR model is that the computational time for a dynamic time-history analysis is reduced from days 
(for running a $\mathrm{FLAC}^{3 \mathrm{D}}$ analysis) to seconds (when performing an OpenSees analysis). Another strength of the NSR model is its ability to simulate foundation force-displacement response, which is mainly affected by $F S v, M /(H D)$ and $\lambda$, by using a unique normalized backbone curve for each of the swaying and rocking degrees of freedom. For foundations resting on an elastic soil deposit having a generalized Gibson stiffness profile as illustrated in Fig. 2, it is common in practice to calculate the foundation stiffness by assuming an equivalent homogeneous soil condition. This is usually done by selecting a representative soil element at an effective depth of the non-homogeneous soil profile such that the stiffness of the foundation on the uniform and non-homogeneous soil deposits is the same [24]. In the case of a yielding foundation, however, two issues arise in determining a uniform soil equivalent for a non-homogeneous soil deposit. Firstly, besides being a function of $\lambda$, the effective depth is also related to FSv. Secondly, the effective depth is obtained by matching only the foundation stiffness while it is irrelevant to the post-yield response and bearing capacity of the foundation, both of which are strongly affected by the moment-to-shear ratio $M /(H D)$. Based on the assumption of a quasi-linear initial foundation stiffness (described in section 4.2.1), a weak equivalence exists for the stated first issue and the effective depth can be calculated using Eqs. (7), (9) and (10) with Figs. 8 and 9. However, this equivalence fails to capture the nonlinear inelastic foundation response when moment and shear capacities are mobilized. On the contrary, in proposed NSR model, the effects of soil non-homogeneity are inherently captured within the adopted normalized backbone curves.

\section{Limitations of NSR Model}

The simplified NSR model is best suited for heavily-loaded structures where uplift is not expected to occur. The model is appropriate for buildings with a small to medium slenderness ratio (i.e. $h_{\text {eff }} / D$ ranging from 0.5-1.25) under strong shaking and was developed for saturated clay conditions. In deriving the global force-displacement response, the nonlinear soil behaviour at small strains is neglected. As the focus of this study is seismic design for strong earthquakes where large strains dominate, it is believed that this feature has negligible impact on the overall behaviour of the soilfoundation-superstructure system. The model is not capable of predicting the settlement of the foundation, however it is capable of capturing the maximum and residual differential settlements (indicated by the rotation $\theta$ ), which are important displacement parameters that influence the design of superstructures.

\section{Conclusions}

A simplified nonlinear sway-rocking model has been developed in this paper for nonlinear dynamic soil-structure interaction analysis. The proposed model is intended to simulate the nonlinear loaddisplacement response for the coupled sway-rocking behaviour of shallow mat foundations supporting heavily-loaded buildings under earthquake ground motions.

To simplify the model, the building is represented as an equivalent SDOF structure, whereas the soilfoundation system is replaced by an assemblage of springs and dashpots. While utilizing independent springs to simulate each of the sway and rocking responses of the foundation, the coupling between the two motions is also accounted for by expressing the spring properties as a function of the load paths experienced by the foundation in the $V: H: M$ space. Spring properties are controlled by the factor of safety against vertical bearing capacity failure $F S v$, the moment-to-shear ratio $M / H$, and the failure envelope defining the bearing capacities of the foundation in the $V: H: M$ 
space. The effect of soil non-homogeneity on the stiffness and capacity of the soil-foundation system is also considered.

In order to identify the load-displacement responses and the coupled bearing capacities of the foundation, a series of static load-control and displacement-control finite-difference analyses were carried out by using the $\mathrm{FLAC}^{3 \mathrm{D}}$ program. The simplified model, developed in the OpenSees platform, was then calibrated against results from the static finite-difference analyses. The effectiveness and efficiency of the proposed model was validated against results from dynamic analyses performed using a $\mathrm{FLAC}^{3 \mathrm{D}}$ model by utilizing two artificial input motions and one real earthquake acceleration record. The comparison of results predicted by both models demonstrates that the simplified model is capable of efficiently capturing the foundation load-displacement behaviour, including the maximum and residual displacements, with sufficient accuracy.

Although the proposed simplified model has some limitations, it is able to provide parameters necessary for preliminary design of buildings on weak soil while achieving a good balance between simplicity and accuracy. In addition, the concept of the model allows engineers to select appropriate model properties in accordance with specific site conditions. 


\section{References}

[1] Gajan S, Kutter BL, Phalen JD, Hutchinson TC, Martin GR. Centrifuge modeling of loaddeformation behavior of rocking shallow foundations. Soil Dyn Earthq Eng 2005;25(7):773-83.

[2] Ugalde JA, Kutter BL, Jeremic B, Gajan S. Centrifuge modeling of rocking behavior of bridges on shallow foundations. Proceedings of the 4 th international conference earthquake geotechnical engineering. Thessaloniki, Greece, Paper2007. p. 25-8.

[3] Anastasopoulos I, Loli M, Georgarakos T, Drosos V. Shaking Table Testing of Rocking-Isolated Bridge Pier on Sand. J Earthq Eng 2013;17(1):1-32.

[4] Gelagoti F, Kourkoulis R, Anastasopoulos I, Gazetas G. Rocking isolation of low-rise frame structures founded on isolated footings. Earthquake Eng Struc 2012;41(7):1177-97.

[5] Chang BJ, Raychowdhury P, Hutchinson TC, Thomas J, Gajan S, Kutter BL. Centrifuge testing of combined frame-wall-foundation structural systems. 8th US National Conference on Earthquake Engineering2006.

[6] Anastasopoulos I, Gazetas G, Loli M, Apostolou M, Gerolymos N. Soil failure can be used for seismic protection of structures. B Earthq Eng 2010;8(2):309-26.

[7] Anastasopoulos I, Gazetas G. Foundation-structure systems over a rupturing normal fault: Part I. Observations after the Kocaeli 1999 earthquake. B Earthq Eng 2007;5(3):253-75.

[8] Figini R, Paolucci R, Chatzigogos CT. A macro-element model for non-linear soil-shallow foundation-structure interaction under seismic loads: theoretical development and experimental validation on large scale tests. Earthquake Eng Struc 2012;41(3):475-93.

[9] Pecker A, Paolucci R, Chatzigogos C, Correia AA, Figini R. The role of non-linear dynamic soilfoundation interaction on the seismic response of structures. B Earthq Eng 2014;12(3):1157-76.

[10] Gazetas G. 4th Ishihara lecture: Soil-foundation-structure systems beyond conventional seismic failure thresholds. Soil Dyn Earthq Eng 2015;68:23-39.

[11] Cremer C, Pecker A, Davenne L. Cyclic macro-element for soil-structure interaction: material and geometrical non-linearities. Int J Numer Anal Met 2001;25(13):1257-84.

[12] Grange S, Kotronis P, Mazars J. A macro-element to simulate dynamic soil-structure interaction. Eng Struct 2009;31(12):3034-46.

[13] Chatzigogos CT, Figini R, Pecker A, Salençon J. A macroelement formulation for shallow foundations on cohesive and frictional soils. Int J Numer Anal Met 2011;35(8):902-31.

[14] Kutter BL, Martin GR, Hutchinson TC, Harden C, Gajan S, Phalen JD. Workshop on modeling of nonlinear cyclic load-deformation behavior of shallow foundations. PEER report no. 2005/14, Pacific Earthquake Engineering Research Center (PEER), University of California, Berkeley 2006. p. 138.

[15] Meek JW, Wolf JP. Cone models for homogeneous soil. I. Journal of geotechnical engineering 1992;118(5):667-85.

[16] Raychowdhury P. Nonlinear winkler-based shallow foundation model for performance assessment of seismically loaded structures: ProQuest; 2008. 
[17] Allotey N, El Naggar MH. An investigation into the Winkler modeling of the cyclic response of rigid footings. Soil Dyn Earthq Eng 2008;28(1):44-57.

[18] Anastasopoulos I, Kontoroupi T. Simplified approximate method for analysis of rocking systems accounting for soil inelasticity and foundation uplifting. Soil Dyn Earthq Eng 2014;56:28-43.

[19] Itasca. FLAC3D: Fast Lagrangian Analysis of Continua in Three Dimensions, Version 5.0. Minneapolis, Minnesota, Itasca Consulting Group 2012.

[20] Gibson RE. Some results concerning displacements and stresses in a non-homogeneous elastic half-space. Geotechnique 1967;17(1):58-67.

[21] Gourvenec S. Failure envelopes for offshore shallow foundations under general loading. Geotechnique 2007;57(9):715-28.

[22] Bell RW. The analysis of offshore foundations subjected to combined loading. MSc, University of Oxford 1991.

[23] Gazetas G, Anastasopoulos I, Adamidis O, Kontoroupi T. Nonlinear rocking stiffness of foundations. Soil Dyn Earthq Eng 2013;47:83-91.

[24] Gazetas G. Static and dynamic displacements of foundations on heterogeneous multilayered soils. Geotechnique 1980;30(2):159-77.

[25] Tan FSC. Centrifuge and theoretical modelling of conical footings on sand. Ph D thesis, Cambridge Univ 1990.

[26] Gourvenec S, Randolph M. Effect of strength non-homogeneity on the shape of failure envelopes for combined loading of strip and circular foundations on clay. Geotechnique 2003;53(6):575-86.

[27] Cassidy MJ, Byrne BW, Houlsby GT. Modelling the behaviour of circular footings under combined loading on loose carbonate sand. Geotechnique 2002;52(10):705-12.

[28] Boulanger RW, Curras CJ, Kutter BL, Wilson DW, Abghari A. Seismic soil-pile-structure interaction experiments and analyses. J Geotech Geoenviron 1999;125(9):750-9.

[29] Gazetas G. Foundation vibrations. Foundation engineering handbook: Springer; 1991. p. 553-93.

[30] OpenSees. Open System for Earthquake Engineering Simulation: OpenSees. Pacific Earthquake Engineering Research Center (PEER), University of California, Berkeley; 2006.

[31] Boulanger RW. The PySimple1, TzSimple1, and QzSimple1 material models, documentation for the OpenSees platform, 2000. http://opensees.berkeley.edu.

[32] Poulos HG, Davis EH. Elastic solutions for soil and rock mechanics: Wiley New York; 1974.

[33] Wong HL, Luco JE. Tables of impedance functions for square foundations on layered media. International Journal of Soil Dynamics and Earthquake Engineering 1985;4(2):64-81.

[34] Waas G, Riggs HR, Werkle H. Displacement solutions for dynamic loads in transversely-isotropic stratified media. Earthquake Eng Struc 1985;13(2):173-93.

[35] Waas G, Hartmann HG, Werkle H. Damping and stiffness of foundations on inhomogeneous media. Proc. 9th World Conf. Earthquake Engng, Tokyo 1988. p. 343-8. 
[36] Hadjian AH, Luco JE. On the importance of layering on impedance functions. Proc. 6th World Conf. Earthquake Engng, New Delhi1977. p. 1680.

[37] Booker JR, Balaam NP, Davis EH. The behaviour of an elastic non-homogeneous half-space. Part II-circular and strip footings. Int J Numer Anal Met 1985;9(4):369-81.

[38] Carrier WD, Christian JT. Rigid circular plate resting on a non-homogenous elastic half-space. Geotechnique 1973;23(1).

[39] Kuhlemeyer RL, Lysmer J. Finite element method accuracy for wave propagation problems. Journal of Soil Mechanics \& Foundations Div 1973;99(Tech Rpt).

[40] Wood DM. Constitutive Modelling. In: Di Prisco C, Wood D, editors. Mechanical Behaviour of Soils Under Environmentally Induced Cyclic Loads: Springer Vienna; 2012. p. 137-226.

[41] Masing G. Eigenspannungen und verfestigung beim messing. Proceedings of the 2nd international congress of applied mechanics: sn; 1926. p. 332-5.

[42] Mejia LH, Dawson EM. Earthquake deconvolution for FLAC. FLAC and numerical modelling in geomechnics, Proceedings of the 4th international FLAC symposium. Madrid, Spain: Itasca Consulting Group; 2006.

[43] Stewart JP, Seed RB, Fenves GL. Empirical evaluation of inertial soil-structure interaction effects: Pacific Earthquake Engineering Research Center; 1998. 